\title{
Avaliação da eficácia agronômica do calcário fosfático no desenvolvimento fenológico
}

\section{e produtivo da cana-de-açúcar}

Evaluation of the agronomic efficacy of phosphatic limestone in phenological and productive development of sugarcane

Evaluación de la eficacia agronómica de la piedra caliza fosfatica en el desarrollo fenológico y productivo de la caña de azúcar

Recebido: 03/06/2021 | Revisado: 14/06/2021 | Aceito: 15/06/2021 | Publicado: 30/06/2021

José Antônio Maior Bono

ORCID: https://orcid.org/0000-0002-9573-9494 Universidade Anhanguera, Brasil E-mail: bono@anhanguera.com

Eduardo Barreto Aguiar

ORCID: https://orcid.org/0000-0002-9525-7559 Universidade Anhanguera, Brasil

E-mail: eduardo.b.aguiar@educadores.net.br

Rhaiany Moreira dos Santos Costa

ORCID: https://orcid.org/0000-0003-4037-8123 Universidade Anhanguera, Brasil E-mail: rhaymoreirac@gmail.com

Flavio Faedo Aguena

ORCID: https://orcid.org/0000-0001-7228-1436 Universidade Anhanguera, Brasil

E-mail: flavioaguenafaedo@gmail.com

Juliano Deimiros Ribeiro

ORCID: https://orcid.org/0000-0002-5102-7153 CropSolutions - Pesquisa, Tecnologia e Inovação Agropecuária Ltda, Brasil E-mail: juliano@cropsolutions.agr.br

Marcos Paulo Martins Corrêa

ORCID: https://orcid.org/0000-0002-0560-7405

Horii agro industrial de minérios Ltda, Brasil E-mail: agromp@live.combr

\section{Resumo}

O objetivo do presente experimento foi avaliar a eficiência de material rocha calcárea com fosfito nos benefícios nutricionais e de fertilidade que podem ser providos pelo calcário dolomítico fosfatado, oriundo do município de Bodoquena/MS, utilizando a cultura da cana-de-açúcar (Saccharum spp) variedade IAC-85-3396, como espécies indicadoras da eficiência nutricional. O estudo foi conduzido em solo classificados como Latossolo Vermelho Distrófico de textura argilosa, e os tratamentos constituídos de 4 doses de calcário $(0,1,2$ e 4 t ha- 1$)$ com dois tipos de calcário, um com fosfito de $4 \%$ de $\mathrm{P} 2 \mathrm{O} 5$ e outro com $8 \% \mathrm{P} 2 \mathrm{O} 5$. Os tratamentos foram distribuídos no campo segundo delineamento experimental de blocos casualisados com 4 repetições. O calcáreo foi aplicado lanço sobre a superfície das parcelas e incorporado na camada de 0 a $20 \mathrm{~cm}$, com gradagem leve e após a niveladora. Os resultados foram submetidos a análise de variância e, ao apresentar diferença significativa, foram submetidos a análise de regressão a $5 \%$ de significância. Os resultados mostraram que os materiais testados foram eficientes como corretivos da acidez do solo, dos níveis de cálcio e magnésio trocáveis no solo e proporcionaram aumento do fósforo disponível às plantas.

Palavras-chave: Calagem; Correção do solo; Desenvolvimento das culturas.

\begin{abstract}
The objective of this experiment was to evaluate the efficiency of calcareous rock material with phosphite in the nutritional and fertility benefits that can be provided by phosphate dolomytic limestone, originating in the municipality of Bodoquena/MS, using sugarcane culture (Saccharum spp) IAC variety-85-3396, as indicator species of nutritional efficiency. The study was conducted in soil classified as Oxisol of clayey texture, and the treatments consisted of 4 doses of limestone $(0,1,2$ and $4 \mathrm{t} / \mathrm{ha}$ ) with two concentrations of phosphite ( $4 \%$ of $\mathrm{P} 2 \mathrm{O} 5$ and $8 \% \mathrm{P} 2 \mathrm{O} 5)$. The treatments were distributed in the field according to an experimental design of randomized blocks with 4 replications. The liming was applied toss on the surface of the plots and incorporated into the layer from 0 to $20 \mathrm{~cm}$, with light gradation and after leveling. The results were submitted to variance analysis and, when presenting significant difference, they were submitted to regression analysis at $5 \%$ significance. The results showed that the materials tested were efficient as
\end{abstract}


correctives of soil acidity, exchangeable calcium and magnesium levels in the soil and provided an increase in the phosphorus available to the plants.

Keywords: Liming; Soil correction; Crop development.

\section{Resumen}

El objetivo de este experimentoera poner en valor la eficiencia del material rupestre calcáreo con fosfito en los beneficios nutricionales y de fertilidad que puede proporcionar la piedra caliza dolomicética de fosfato, originaria del municipio de Bodoquena/EM, utilizando la variedad IAC-85-3396 del cultivo de caña de azúcar (Saccharumspp), como especie indicadora de eficiencia nutricional. El estudio se llevó a cabo en suelos clasificados como Latosol Roja Distrófica de textura arcillosa, y los tratamientos consistieron en 4 dosis de piedra caliza $(0,1,2$ y 4 tha- 1$)$ con dos concentraciones de fosfito (4\% de P2O5 y $8 \%$ P2O5). Los tratamientos se distribuyeron en el campo de acuerdo con un diseño experimental de bloques aleatorizados con 4 replicaciones. El calcáreo se aplicó en la superficie de las parcelas y se incorporó a la capa de 0 a $20 \mathrm{~cm}$, con gradación de luz y después de la nivelación. Los resultados se sometieron al análisis de desviaciones $\mathrm{y}$, al presentar diferencias significativas, se sometieron a un análisis de regresión a una importancia del $5 \%$ significativos. Los resultados mostraron que los materiales probados eran eficientes como correctivos de acidez del suelo, calcio intercambiable y niveles de magnesio en el suelo y proporcionaban un aumento del fósforo disponible para las plantas.

Palabras clave: Cátodo; Corrección del suelo; Desarrollo de cultivos.

\section{Introdução}

A prática difundida na agricultura brasileira e correção da acidez solo, sendo para tanto utilizado rochas carbonáticas, visando a elevação do $\mathrm{pH}$, alterando a disponibilidade de nutrientes no solo para as plantas, além do fornecimento de $\mathrm{Ca}$ e $\mathrm{Mg}$ trocáveis e influenciando diretamente o alumínio disponível, tóxicos as plantas e a saturação por bases do solo (Pavinato \& Rosolem, 2008; Soratto, et al., 2010).

Souza, et al., 2007) relatam ainda que eliminação da acidez do solo aumento da disponibilidade de fósforo, redução da disponibilidade do manganês tóxico, através da formação de hidróxidos, aumento da mineralização da matéria orgânica e favorecimento da fixação biológica de nitrogênio.

O fósforo, juntamente com o cálcio, influencia no crescimento radicular, tornando, assim, elementos fundamentais nas construções da fertilidade do solo ao longo do perfil do solo, importante para a cultura da cana-de-açúcar, para um desenvolvimento radicular. Aquino, et al., 2015, comentam que o sistema radicular da cana de açúcar representa um importante, pois desempenha papel essencial na regeneração das soqueiras, após a colheita, e influencia diretamente a eficiência de absorção dos nutrientes pela planta, a resistência à seca e a tolerância ao ataque de pragas do solo, o que afeta todo o desenvolvimento da cultura.

O uso de corretivos de solo visando a dessorção do íon fosfato, minimiza a retenção do elemento aplicado, aumentando a disponibilidade para as plantas, dessa forma, é possível tornar solos de baixa fertilidade natural, produtivos (Malavolta, 1980).

Lopes (1984) apontou a necessidade de calagem para elevar o $\mathrm{pH}$ dos solos de Cerrado a valores acima de 5,5 para ativar a formação de cargas negativas na fração orgânica do solo, aumentar a capacidade de troca catiônica e reduzir o potencial de perdas de cátions por lixiviação. Cálcio, magnésio e potássio são elementos que afetam o complexo de troca de cátions no solo e podem representar até $90 \%$ do poder de troca de íons no solo (Vitti, et al., 2006).

O fósforo ocorrente no solo, de interesse agronômico ou ambiental, é constituído por compostos derivados do ácido ortofosfórico e, menos comumente, dos pirofosfatos. Os minerais primários fosfatados comuns em rochas como as apatitas, de onde são liberados durante a intemperização, resultando em minerais secundários mais estáveis termodinamicamente, ou incorporados a compostos orgânicos biologicamente.

A dinâmica do fósforo no solo está associada a fatores ambientais que controlam a atividade dos microrganismos, os quais imobilizam ou liberam os íons ortofosfato, e às propriedades físico-químicas e mineralógicas do solo. Assim, em solos jovens e nos moderadamente intemperizados, como os Vertissolos, Chernossolos e os Neossolos, ainda ocorre fósforo em minerais primários, mas a maior parte deste elemento se encontra na forma orgânica (Po), ou na forma mineral (Pi), adsorvida 
fracamente aos minerais secundários. Nos solos altamente intemperizados, como os Latossolos, predominam as formas inorgânicas ligadas à fração mineral com alta energia e as formas orgânicas estabilizadas física e quimicamente. De acordo com o maior ou menor grau de estabilidade destes compostos, são enquadrados como fosfatos lábeis e não-lábeis. A fração lábil é representada pelo conjunto de compostos fosfatados capazes de repor rapidamente a solução do solo, quando ele é absorvido por plantas ou por microrganismos. Por isso, as frações mais lábeis são dependentes do grau de intemperização do solo, da mineralogia, da textura, do teor de matéria orgânica, das características físico-químicas, da atividade biológica e da vegetação predominante (Walker \& Syers, 1976; Cross \& Schlesinger, 1995).

A maioria das fontes corretivas de acidez no Brasil, rochas dolomíticas e ou calcificas, não apresenta fósforo na sua composição, assim a fonte de fósforo é através de fertilizantes fosfatados. Esta prática formas inorgânicas de fósforo no solo é a mais pronunciada, com diferentes graus de energia de ligação (Rheinheimer \& Anghinoni, 2001), provocando assim a adsorção do fósforo sítios mais ávidos (de menor labilidade) (Rheinheimer \& Anghinoni, 2006).

Neste contexto, um corretivo da acidez que fornece simultaneamente o fósforo $(\mathrm{P})$ de rocha origem alcalina, traria benefícios para atividade agrícola, reduzindo os custos com a adubação fosfata e a melhoria da eficiência de absorção do nutriente pelas plantas. Justos (1999) evidenciou na região a presença de rochas carbonatas com fosforito, ou seja, a possibilidade de produção de calcáreo com altos teores de fósforo, podendo assim tornar um importante insumo para uso na agricultura. Além de corretivo da acidez e levar os nutrientes $\mathrm{Ca}$ e $\mathrm{Mg}$, poderá fornecer expressiva quantidade de fósforo ao solo, simultaneamente.

O Objetivo deste trabalho foi avaliar a eficiência de material rocha calcáreo dolomítico com fosfito na fertilidade do solo e na nutrição de planta, oriundo do município de Bodoquena/MS, utilizando a cultura da cana-de-açúcar (Saccharum spp), como espécies indicadoras da eficiência nutricional.

\section{Metodologia}

O local experimental situa-se em clima classificado como Cwa, segundo a classificação de Köppen e o solo como Latossolo Vermelho distrófico, textura média. A localização do estudo foi nas coordenadas de Latitude: $20^{\circ} 26^{\prime} 34^{\prime \prime}$ 'S e Longitude: $54^{\circ} 38^{\prime} 47^{\prime \prime} \mathrm{O}$ com altitude de 540 metros. Na Tabela 1, observamos as a análise química e física do solo, conforme Embrapa (2011).

Tabela 1. Análise química do solo coletado no local do ensaio com a cultura da cana-de-açúcar (Saccharum spp).

\begin{tabular}{|c|c|c|c|c|c|c|c|c|c|c|c|c|c|c|}
\hline \multirow{2}{*}{$\begin{array}{l}\mathrm{pH} \\
\text { água }\end{array}$} & \multirow{2}{*}{$\begin{array}{l}\mathrm{pH} \\
\mathrm{CaCl}_{2}\end{array}$} & \multirow[b]{2}{*}{--} & $\mathrm{K}$ & $\mathrm{Ca}$ & $\mathrm{Mg}$ & $\mathrm{H}$ & Al & M.O & SB & $\begin{array}{l}\text { CTC } \\
\mathrm{pH} 7,0\end{array}$ & \multirow{2}{*}{$\begin{array}{l}\mathrm{V} \\
\%\end{array}$} & \multirow{2}{*}{\multicolumn{2}{|c|}{$\begin{array}{c}\text { Argila Silte } \\
\text {-------- } \mathrm{g} \mathrm{kg}^{-1}\end{array}$}} & \multirow{2}{*}{ Areia } \\
\hline & & & $---\mathrm{mg} \mathrm{dm}{ }^{-3}---$ & \multicolumn{3}{|c|}{---- cmol dm $\mathrm{dm}^{-3}$----- } & \multicolumn{2}{|c|}{----- $\mathrm{dm}^{-3}$} & \multicolumn{2}{|c|}{$-\mathrm{cmol} \mathrm{dm}{ }^{-3}---$} & & & & \\
\hline
\end{tabular}

\footnotetext{
M.O.: matéria orgânica; P: fósforo; K: potássio; Ca: cálcio; Mg: magnésio; Al: alumínio; H: hidrogênio; SB: soma de bases; CTC: capacidade de troca de cátions; $\mathrm{V} \%$ : porcentagem por saturação de base.

Fonte: Autores
}

Os tratamentos foram constituídos de um fatorial com doses de calcáreo $(0,1,2$, e 4 t/ha) e duas concentrações de fosfito na rocha $\left(4 \% \mathrm{P}_{2} \mathrm{O}_{5}\right.$ e $\left.8 \% \mathrm{P}_{2} \mathrm{O}_{5}\right)$. O solo foi preparado com gradagem, distribuição do calcáreo foi à lanço e incorporado com a enxada rotativa na camada de 0 a $20 \mathrm{~cm}, 3$ meses antes do transplante das mudas de cana. As parcelas experimentais com dimensões de 5,6 x 5,0 m por parcela. Os tratamentos foram distribuídos, nas parcelas experimentais, segundo o delineamento de blocos ao acaso com 4 repetições.

O transplante das mudas (MPB) foi no dia em outubro de 2019, com espaçamento de 1,4 m entre linhas e 0,6 m entre as mudas da variedade IAC-85-3396, produzidas pelo sistema de mudas pré-brotadas (MPB), desenvolvido pelo Instituto 
Agronômico de Campinas (IAC). As mudas foram produzidas no período de março a setembro de 2019, sendo os minis toletes foram obtidos de um viveiro primário de uma usina de açúcar e álcool da região. Os mesmos foram colocados para germinar (brotar) em estufa incubadora BOD a $33^{\circ} \mathrm{C}$ por 3 dias e após transferidos para tubetes e mantido em cada de vegetação com irrigação automatizadas duas vezes ao dia.

O produto utilizado para o estudo foi obtido diretamente de uma mineradora (Mineradora Horii) localizada no município de Bodoquena. O material foi moído de acordo com as normas do Ministério da Agricultura e Abastecimento (MAPA) para corretivos e submetido a análise para caracterização mineralógica por difração de raio-X (Brasil, 2014). O material moído passo $100 \%$ na peneira de $<0,3 \mathrm{~mm}$, ou seja, sua eficiência relativa (ER) foi de $100 \%$. Na Tabela 2 estão os valores da análise, conforme o MAPA para a caracterização do material utilizados no estudo.

Tabela 2. Valores obtidos da análise dos materiais utilizados no experimento.

\begin{tabular}{|c|c|c|c|c|c|c|c|c|c|c|c|c|}
\hline $\mathrm{K}_{2} \mathrm{O}$ & $\mathrm{Cu}$ & $\mathrm{Fe}$ & $\mathrm{Mn}$ & $\mathrm{Zn}$ & $\mathrm{S}$ & $\mathrm{Co}$ & Mo & $\mathrm{CaO}$ & $\mathrm{MgO}$ & $\mathrm{PN}$ & $\mathrm{SiO}_{2}$ & $\mathrm{Cl}$ \\
\hline & \multicolumn{12}{|c|}{ Calcário com $4 \%$ de $\mathrm{P}_{2} \mathrm{O}_{5}$} \\
\hline $0,13 \pm$ & $0,01 \pm$ & $0,33 \pm$ & $0,02 \pm$ & $0,01 \pm$ & $0,10 \pm$ & $0,14 \pm$ & $0,11 \pm$ & $37,6 \pm$ & $14,1 \pm$ & $87,3 \pm$ & $8,4 \pm$ & $0,1 \pm$ \\
\hline 0,05 & 0,002 & 0,03 & 0,002 & 0,003 & 0,04 & 0,06 & 0,005 & 2,5 & 1,7 & 6,3 & 1,2 & 0,05 \\
\hline
\end{tabular}

\section{Calcário com $8 \%$ de $\mathrm{P}_{2} \mathrm{O}_{5}$}

\begin{tabular}{|c|c|c|c|c|c|c|c|c|c|c|c|c|}
\hline $0,1 \pm$ & $0,01 \pm$ & $0,74 \pm$ & $0,05 \pm$ & $0,01 \pm$ & $0,11 \pm$ & $0,09 \pm$ & $0,11 \pm$ & $33,3 \pm$ & $10,6 \pm$ & $57,8 \pm$ & $22,6 \pm$ & $0,08 \pm$ \\
\hline 0,03 & 0,002 & 0,08 & 0,002 & 0,005 & 0,03 & 0,002 & 0,03 & 2,35 & 1,23 & 8,65 & 6,32 & 0,002 \\
\hline
\end{tabular}

$\mathrm{K}_{2} \mathrm{O}$ : Óxido de Potássio; Cu: Cobre; Fe: Ferro; Mn: Manganês; Zn: Zinco; S: Enxofre; Co: Cobalto; Mo: Molibdênio ; $\mathrm{P}_{2} \mathrm{O} \%$ : Óxido de Fósforo; CaO: Óxido de Cálcio; MgO: Óxido de Magnésio; PN: Poder neutralizante ; SiO: Óxido de Silício; Cl: Cloro.

Fonte: Autores.

Após 4 meses do transplante das mudas, coletou-se a lâmina foliar da folha +3 , totalizando 30 folhas por parcela experimental. As folhas foram secas em estufa a $65^{\circ} \mathrm{C}$ com circulação de ar forçada e posteriormente moídas em moinho tipo Wille e analisados os teores foliares de N, P, K, Ca, Mg, S, Zn, Cu, Mn, B e Mo, conforme Embrapa (2009).

Aos 387 dias após o transplante (outubro de 2020), efetuou-se o corte dos colmos na parcela útil, cortando-os rente ao solo, despontando a parte do palmito e pesando os mesmos. Efetuou-se a contagem dos perfilhos da cana-de-açúcar e altura de planta, adotando como referência a superfície do solo até a folha +1 ( $1^{\mathrm{a}}$ folha com lígula visível). Após o corte determinou-se o grau Brix através de refratômetro portátil, medindo a parte superior do colmo e a base e registrando o valor médio obtido, em dez colmos por parcela. Após procedeu a coleta das amostras de solo, utilizando o trado sonda, nas camadas de 0 a $20 \mathrm{~cm}$ e 20 a $40 \mathrm{~cm}$ e analisadas para avaliação da fertilidade do solo, sendo o fósforo avaliado pelos extratores Mehlich-1 e Resina trocadoras ânions, conforme Embrapa (2011).

Os dados foram submetidos à análise de variância (Teste F) e quando significativo os dados foram submetidos a análise de regressão a 5\% de significância.

\section{Resultados e Discussão}

O perfilhamento e altura das plantas, foram influenciados pelas doses de calcário e não apresentando diferenças significativas para os tipos de calcário. Utilizando os modelos matemáticos para a curva de resposta verifica-se que os pontos de máxima resposta foi na dose de $3,76 \mathrm{t} \mathrm{ha}^{-1}$ e $2,54 \mathrm{t} \mathrm{ha}^{-1}$, para o perfilhamento e 2,9 $\mathrm{t} \mathrm{ha}^{-1} \mathrm{e} 2,8 \mathrm{t} \mathrm{ha}^{-1}$ para altura das plantas, 
respectivamente para os calcários com $4 \% \mathrm{P}_{2} \mathrm{O}_{5}$ e $8 \% \mathrm{P}_{2} \mathrm{O}_{5}$, indicando que os tipos de calcário tiveram influência no perfilhamento da planta. Na máxima resposta das doses de calcário, o número de perfilhos foram de 14,5 no de $4 \% \mathrm{P}_{2} \mathrm{O}_{5}$ e de 13,0 no de $8 \% \mathrm{P}_{2} \mathrm{O}_{5}$ e altura de planta de $2,5 \mathrm{~m}$ e 2,7 m, respectivamente para os calcários $4 \% \mathrm{P}_{2} \mathrm{O}_{5}$ e $8 \% \mathrm{P}_{2} \mathrm{O}_{5}$ (Figura 1).

Figura 1. Valores médios de números de perfilho por planta e altura do colmo da cana-de-açúcar cultivada em solo corrigido com calcário fosfático.
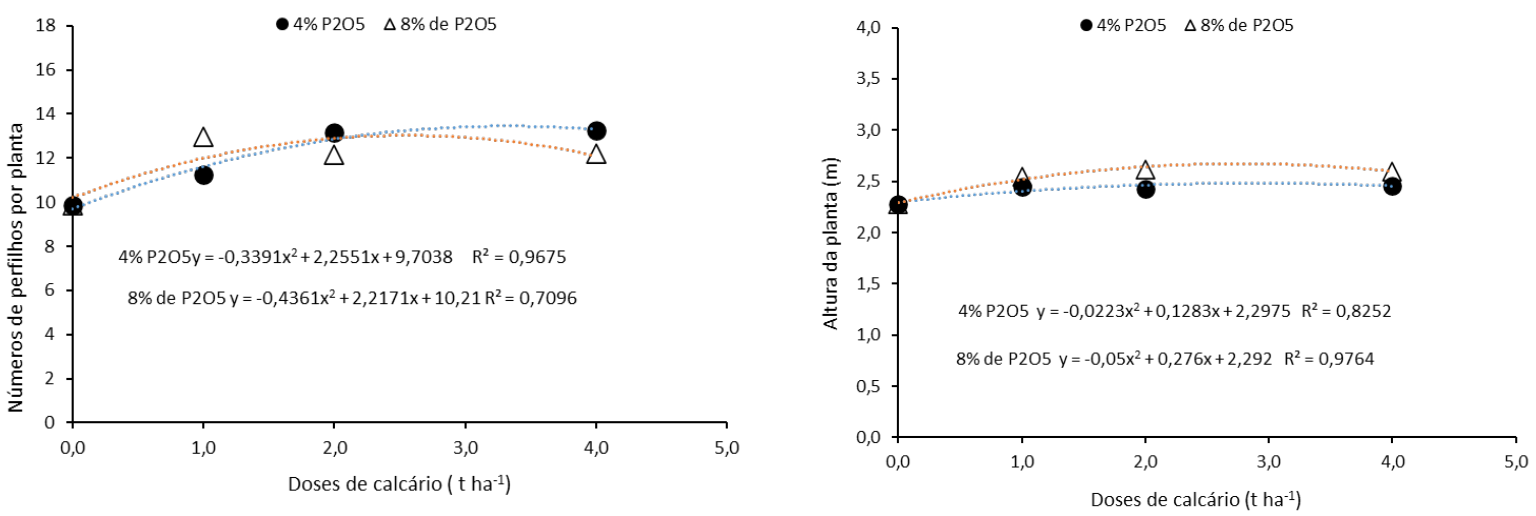

Fonte: Autores.

O calcário de $4 \% \mathrm{P}_{2} \mathrm{O}_{5}$, proporcionou maior perfilhamento, no entanto, ficou com altura de colmos inferior ao de $8 \%$ $\mathrm{P}_{2} \mathrm{O}_{5}$. Tavares et al (2010) verificaram altura de colmos aos 457 dias após o plantio de próximo a 2,5 m, valor semelhante ao encontrado neste trabalho. Silva et al (2008) trabalhando com os cultivares IAC86-2480 e RB72-454 observaram respectivamente 12,0 e 10,99 perfilhos, valores inferiores ao encontrado neste estudo.

O maior perfilhamento no calcário $4 \% \mathrm{P}_{2} \mathrm{O}_{5}$, pode estar relacionado ao menor crescimento da planta, pode está associado ao efeito das auxinas. O fluxo de auxinas do ápice para base é diminuído e, consequentemente, haveria decréscimo do grau de inibição das gemas laterais, resultando na formação de mais perfilhos. Jadoski, et al. (2010) comentam que a o efeito do fluxo de auxina, isso é, se diminuir e reduz a inibição das gemas laterais favorecendo, assim o perfilhamento. Como as plantas no calcário $8 \% \mathrm{P}_{2} \mathrm{O}_{5}$, cresceram um pouco mais, elas podem ter um efeito mais pronunciado na produção das auxinas no ápice e consequente, reduzindo um pouco o perfilhamento.

O maior perfilhamento no calcário com $8 \% \mathrm{P}_{2} \mathrm{O}_{5}$, pode estar associado também relacionado ao $\mathrm{SiO}_{2}$, mais concentrado neste material em relação ao outo (Tabela 2). Silveira Junior, et al. (2003) relatam que acumulação de Si em plantas como a cana-de-açúcar pode redução da transpiração contribuindo substancialmente para fortalecer a estrutura da planta e aumentar a resistência ao acamamento. Neste caso, uma planta mais ereta o efeito inibidor das auxinas pode ter sido maior neste material, e reduzindo o perfilhamento.

Embora os materiais utilizados como corretivos, apresenta-se com vários elementos nutrientes na sua composição, como $\mathrm{Cu}, \mathrm{Fe}, \mathrm{Mn}, \mathrm{Zn}, \mathrm{K}$, e principalmente $\mathrm{P}, \mathrm{Ca}$ e $\mathrm{Mg}$ (Tabela 2), não influenciaram na nutrição de plantas, quando avaliado pelos teores foliares (Tabela 3), com e exceção do nutriente fósforo (Figura 2). 
Tabela 3. Valores médios dos teores foliares da cana-de-açúcar cultivada em solo corrigido com calcário fosfático.

\begin{tabular}{|c|c|c|c|c|c|c|c|c|c|c|c|}
\hline Trat. & $\mathrm{N}$ & $\mathrm{P}$ & $\mathrm{K}$ & $\mathrm{Ca}$ & $\mathrm{Mg}$ & $\mathrm{S}$ & $\mathrm{Zn}$ & $\mathrm{Cu}$ & $\mathrm{Fe}$ & $\mathrm{Mn}$ & B \\
\hline $\mathrm{tha}^{-1}$ & --------- & --1--- & $--\mathrm{g} \mathrm{kg}^{-1}-\ldots$ & - & 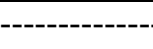 & & -- & ------- & $--\mathrm{mg} \mathrm{kg}^{-1}$ & - & \\
\hline 0 & $16,3 \pm 1,21$ & $1,2 \pm 0,08$ & $12,5 \pm 0,6$ & $4,3 \pm 0,2$ & $2,0 \pm 0,06$ & $1,5 \pm 0,02$ & $7,4 \pm 0,5$ & $6,1 \pm 0,9$ & $84,1 \pm 6,3$ & $194,5 \pm 16,3$ & $11,7 \pm 0,96$ \\
\hline & \multicolumn{11}{|c|}{ Calcário - $4 \% \mathrm{P}_{2} \mathrm{O}_{5}$} \\
\hline 1 & $17,9 \pm 1,24$ & $\begin{array}{c}1,3 \pm \\
0,07\end{array}$ & $11,8 \pm 0,8$ & $4,8 \pm 0,3$ & $2,3 \pm 0,04$ & $1,5 \pm 0,03$ & $7,0 \pm 0,8$ & $7,0 \pm 0,6$ & $108,7 \pm 7,1$ & $213,9 \pm 18,2$ & $13,1 \pm 1,05$ \\
\hline 2 & $16,9 \pm 1,22$ & $\begin{array}{c}1,4 \pm \\
0,05\end{array}$ & $12,8 \pm 0,7$ & $4,4 \pm 0,4$ & $2,0 \pm 0,06$ & $1,4 \pm 0,06$ & $6,7 \pm 0,9$ & $6,4 \pm 0,5$ & $76,5 \pm 6,5$ & $240,1 \pm 21,7$ & $12,8 \pm 1,07$ \\
\hline 4 & $18,2 \pm 1,09$ & $\begin{array}{c}1,5 \pm \\
0,06\end{array}$ & $12,7 \pm 0,9$ & $4,5 \pm 0,3$ & $2,3 \pm 0,09$ & $1,4 \pm 0,04$ & $6,9 \pm 1,1$ & $7,3 \pm 0,7$ & $98,6 \pm 10,3$ & $235,2 \pm 16,4$ & $13,5 \pm 1,11$ \\
\hline \multirow[t]{2}{*}{ Média } & $17,7 \mathrm{a}$ & $1,4 \mathrm{~b}$ & $12,4 \mathrm{a}$ & $4,6 \mathrm{a}$ & $2,2 \mathrm{a}$ & $1,4 \mathrm{a}$ & $6,9 \mathrm{a}$ & $6,9 \mathrm{a}$ & $94,6 \mathrm{a}$ & $229,7 \mathrm{a}$ & $13,1 \mathrm{a}$ \\
\hline & \multicolumn{11}{|c|}{ Calcário - $8 \% \mathrm{P}_{2} \mathrm{O}_{5}$} \\
\hline 1 & $18,4 \pm 1,19$ & $1,5 \pm 0,03$ & $14,2 \pm 1,05$ & $4,2 \pm 0,2$ & $2,1 \pm 0,04$ & $1,3 \pm 0,05$ & $7,8 \pm 0,6$ & $6,1 \pm 0,09$ & $65,6 \pm 8,3$ & $187,2 \pm 16,4$ & $13,7 \pm 1,09$ \\
\hline 2 & $17,1 \pm 0,96$ & $1,6 \pm 0,08$ & $12,9 \pm 1,02$ & $4,5 \pm 0,4$ & $1,9 \pm 0,06$ & $1,3 \pm 0,02$ & $7,5 \pm 0,8$ & $5,6 \pm 0,03$ & $58,8 \pm 5,6$ & $226,3 \pm 15,2$ & $13,8 \pm 0,87$ \\
\hline 4 & $17,6 \pm 1,12$ & $1,9 \pm 0,07$ & $13,2 \pm 0,96$ & $4,7 \pm 0,1$ & $2,0 \pm 0,05$ & $1,4 \pm 0,03$ & $7,6 \pm 1,03$ & $5,7 \pm 0,05$ & $63,5 \pm 7,3$ & $215,8 \pm 17,2$ & $13,5 \pm 1,24$ \\
\hline Média & $17,7 \mathrm{a}$ & $1,7 \mathrm{a}$ & $13,4 \mathrm{a}$ & $4,5 \mathrm{a}$ & $2,1 \mathrm{a}$ & $1,3 \mathrm{a}$ & $7,6 \mathrm{a}$ & $5,8 \mathrm{a}$ & $62,6 \mathrm{a}$ & $209,8 \mathrm{a}$ & $13,7 \mathrm{a}$ \\
\hline
\end{tabular}

Letras diferentes na coluna diferente entre pelo teste $\mathrm{F}$ a $5 \%$ probabilidade.

Fonte: Autores.

Figura 2. Valores médios dos teores de fósforo na lâmina foliar da cana-de-açúcar cultivada em solo corrigido com calcário fosfático. * e ** significativo a $5 \%$ e $1 \%$ de probabilidade, respectivamente.

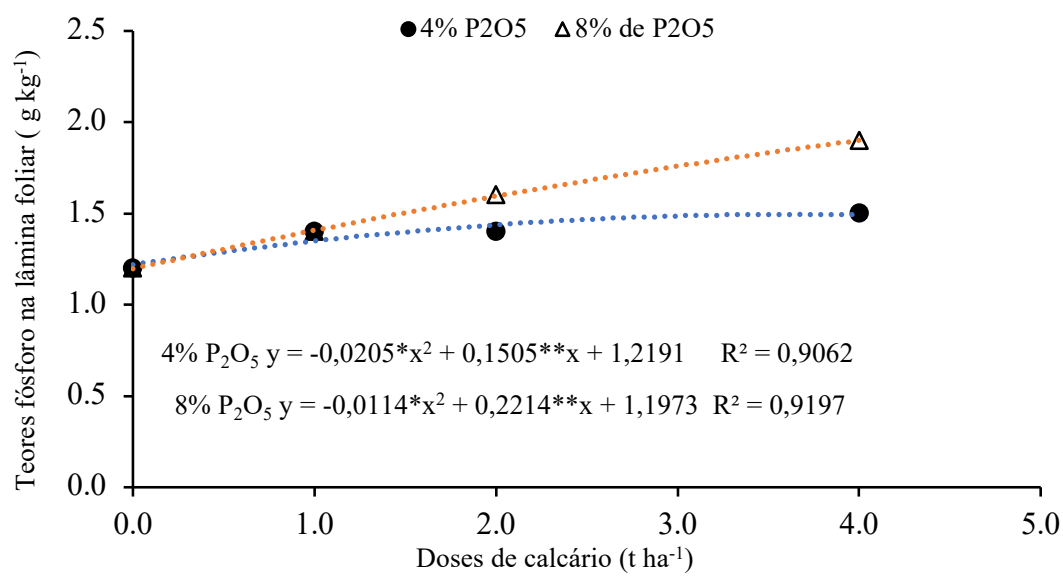

Fonte: Autores.

As concentrações dos nutrientes nos matérias corretivos de $\mathrm{Cu}, \mathrm{Fe}, \mathrm{Mn}, \mathrm{Zn}, \mathrm{K}$, são muito baixas e não tiveram efeito nutricional durante o período do estudo. No entanto, os teores de $\mathrm{Ca}$ e $\mathrm{Mg}$ são altos e não influenciaram de maneira significativa os teores foliares. Este fato, pode ser devido que estes elementos químicos estarem próximos dos níveis críticos no solo. Alvares V. et al. (1999) estabelece os valores de 2,4 e $0,9 \mathrm{cmol}_{+} \mathrm{dm}^{-3}$, respectivamente para Ca e $\mathrm{Mg}$ como níveis críticos no solo. No solo utilizado para o estudo verificamos os valores de $2,3 \mathrm{cmol}_{+} \mathrm{dm}^{-3}$ para Ca e 1,2 $\mathrm{cmol}_{+} \mathrm{dm}^{-3}$ para o Mg (Tabela 1), portanto, 
estes nutrientes já estariam no limite e acima dos níveis críticos para a cultura. Já, para o fósforo o valor no solo de $2,0 \mathrm{mg} \mathrm{dm}^{-3}$ (Tabela 1) está muito abaixo do seu nível crítico de $20 \mathrm{mg} \mathrm{dm}^{-3}$, segundo Alvarez V. et al. (1999).

A maior concentração de fósforo foliar foi a partir de $2 \mathrm{t} \mathrm{ha}^{-1}$ de calcário, ou seja, doses abaixo destes valores pouco efeito nutricional deste elemento para a cultura. Considerando a concentração de $8 \%$ de $\mathrm{P}_{2} \mathrm{O}_{5}$, no material corretivo, assim, levaria 80,160 e $320 \mathrm{~kg} \mathrm{ha}^{-1} \mathrm{de}_{2} \mathrm{O}_{5}$, nas respectivas doses de $1,2 \mathrm{e} 4 \mathrm{tha}^{-1}$, serem quantidades expressivas e podem ao longo do tempo consideram esta operação com uma fosfatagem para a cultura. Silva et al. (2019) trabalhando com na cana e com fosfato natural reativo, verificaram que na dose $150 \mathrm{~kg} \mathrm{ha}^{-1} \mathrm{de}_{2} \mathrm{O}_{5}$ reduziu a atividade das enzimas antioxidantes e aumentou em 25,0\% a biomassa seca da parte aérea. A fonte utilizada como corretivo e de rocha sedimentar e, portanto, o fósforo associado a rocha é de origem alcalina. Souza, et al. (2014) verificaram a eficiência agronômica do fosfato natural de Bayóvar, solo com elevado teor de Ca trocável e pH 6, chegou a faixa de 72 a $82 \%$ de eficiência agronômica, indicando que uso de fonte naturais de fosforo pode ser utilizada ambiente corrigido.

Os calcários utilizados proporcionaram aumento na produtividade de colmos, independentemente do tipo e não tiveram efeito significativo nos teores de BRIX (Figura 3).

Figura 3. Produtividade de colmos de cana no primeiro ano, submetidos a doses de calcário fosfático. ${ }^{*} \mathrm{e} * *$ significativo a $5 \%$ e $1 \%$ de probabilidade, respectivamente, ${ }^{\text {ns }}$ não significativo.

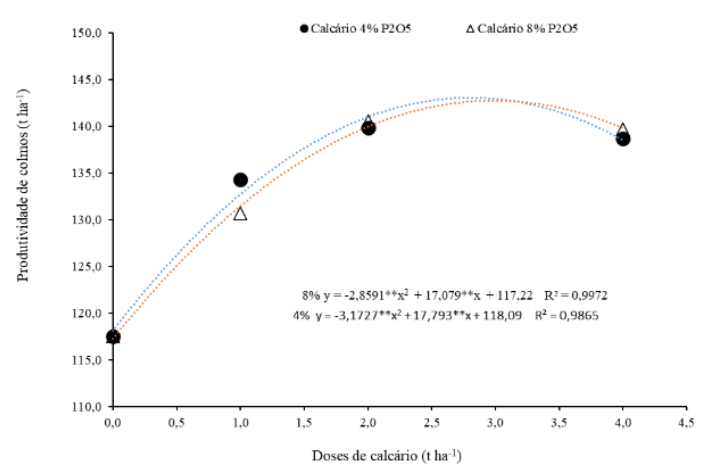

Fonte: Autores.

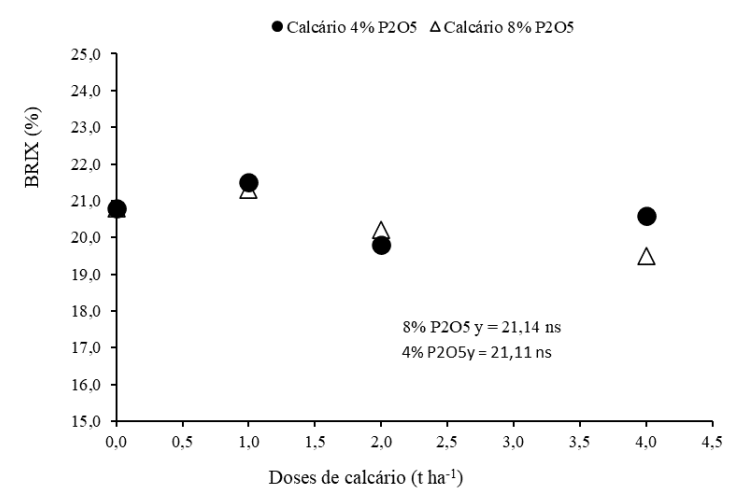

O calcário de $4 \%$ de $\mathrm{P}_{2} \mathrm{O}_{5}$, apresentou máximo reposta a 2,80 tha ${ }^{-1}$, de acordo com o modelo matemático ajustado, correspondendo a produtividade de $143,03 \mathrm{t} \mathrm{ha}^{-1}$ de colmos de cana. Para o calcário de $8 \%$ de $\mathrm{P}_{2} \mathrm{O}_{5}$, a máxima resposta foi de 2,98 $\mathrm{t} \mathrm{ha}^{-1}$, o que daria uma produtividade de 142,73 $\mathrm{t} \mathrm{ha}^{-1}$. Os calcários de 4 e $8 \%$ de $\mathrm{P}_{2} \mathrm{O}_{5}$ apresentaram 21,72\% e 21,47\%, a mais de produtividade em relação a testemunha, respectivamente. Isso indica que os produtos promovem aumento de produtividade na cana, no primeiro ano de cultivo. Bambolim et al (2015) comentam que a correçãoda acidez é necessária par a sustentabilidade para não comprometer o desenvolvimento da cultra e propiciar aumento na produtividade, fato comprovado pelo materiais calcarios utilizados no trabalho. Estes resultados, corroboram com os de por Ferraz, et al. (2015). Zandona, et al. (2015), reforça ainda que a calagem favorece o cescimento radicular e a absorção de água e nutrientes, o que também pode a explicar o aumento da produtividade nas doses de calcário utilizadas, neste estudo.

A melhoria quimica do solo, com o uso dos corretivos, é evidenciado nos valores de pH da solução do solo (Figura 4). 
Figura 4. Valores do pH da solução do solo (em água e $\mathrm{CaCl}_{2}$ ), para as doses de calcário. * e ** significativo a 5\% e $1 \%$ de probabilidade, respectivamente.
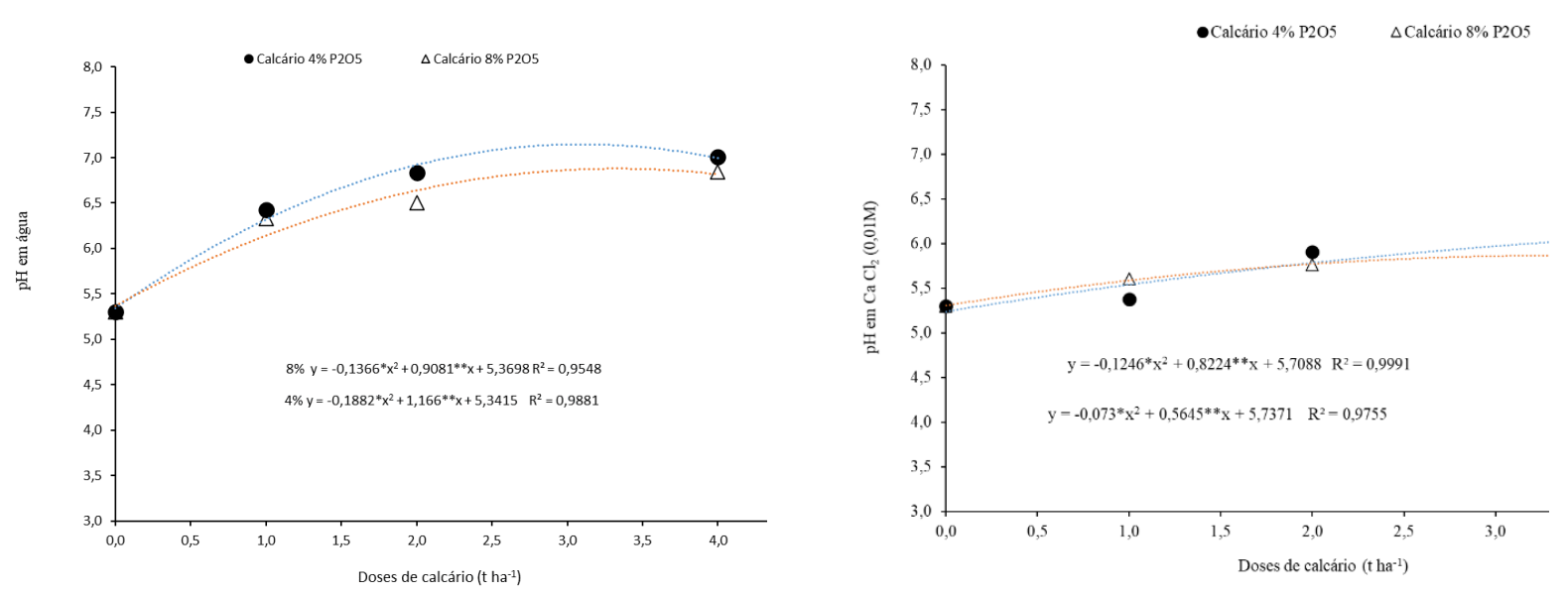

Fonte: Autores.

Pelo ajuste do modelo matemático, o pH em água teve sua máxima resposta na dosagem de 3,08 e 3,32 $\mathrm{t} \mathrm{ha}^{-1}$, com valores de pH em água de 7,2 e 6,9, respectivamente para calcários com $4 \%$ e $8 \%$ de $\mathrm{P}_{2} \mathrm{O}_{5}$, evidenciando o efeito corretivo dos materiais testados quando comparados com o tratamento testemunha $(5,3)$. No $\mathrm{pH}$ em água o calcário com $4 \%$ de $\mathrm{P}_{2} \mathrm{O}_{5}$ apresentou valores supriores aos de $8 \%$. Este fato pode estar associado ao PRNT do calcário maior no de $4 \%$ estava com PRNT de $87,3 \%$ e no de $8 \%$ com PRNT de 57,8\%. Este fato, não ficou evidente no $\mathrm{pH}$ em $\mathrm{CaCl}_{2}$.

Para o $\mathrm{pH}$ em $\mathrm{CaCl}_{2}$, observou a mesma tendência, com exceção do calcário com $4 \%$ de $\mathrm{P}_{2} \mathrm{O}_{5}$, em que o ajuste do modelo matemático foi linear. Este ajuste linear indica que o calcário ainda estaria reagindo no solo e não atingiu seu ponto de máxima resposta, no entanto, o periodo entre a aplicação e coleta de amostra de solo foram de 457 dias, tempo sufuciente para que os materiais pudessem reagir.

Para o $\mathrm{pH}$ em $\mathrm{CaCl}_{2}$, o calcário com $8 \% \mathrm{P}_{2} \mathrm{O}_{5}$ apresentou a máxima resposta com aplicação de $3,38 \mathrm{t} \mathrm{ha}^{-1} \mathrm{e}$ valor de $\mathrm{pH}$ da solução do solo em 5,9, evidenciando também efeito corretivo, quando comparado com o tratamento testemunha $(4,8)$.

A elevação dos valores de $\mathrm{pH}$ da solução do solo, infleunciou a disponibilidade de fósforo. A máxima resposta da disponibilidade de fósforo (P) foi de 3,74 e 3,27 t ha ${ }^{-1}$, para o calcário com 4 e $8 \%$ de $\mathrm{P}_{2} \mathrm{O}_{5}$, respectivamente, para o extrator Mehlich-1. Para a Resina, a máxima respota foi de 3,64 t ha ${ }^{-1}$ para o calcário de $8 \%$ de $\mathrm{P}_{2} \mathrm{O}_{5}$, sendo que o calcário de $4 \% \mathrm{P}_{2} \mathrm{O}_{5}$ teve um ajuste de modelo linear (Figura 5). O aumento da disponibilidade de fosforo para as plantas, tanto pelo Mehlich quanto pela Resina, indica que os materias testados tem potencial em corrigir as deficiências de fósforo no solo. 
Figura 5. Fósforo disponível no solo (pelos extratores Mehlich-1 e Resina), com doses de calcário fosfático. * e ** significativo a $5 \%$ e $1 \%$ de probabilidade, respectivamente. ${ }^{\text {ns }}$ não significativo.
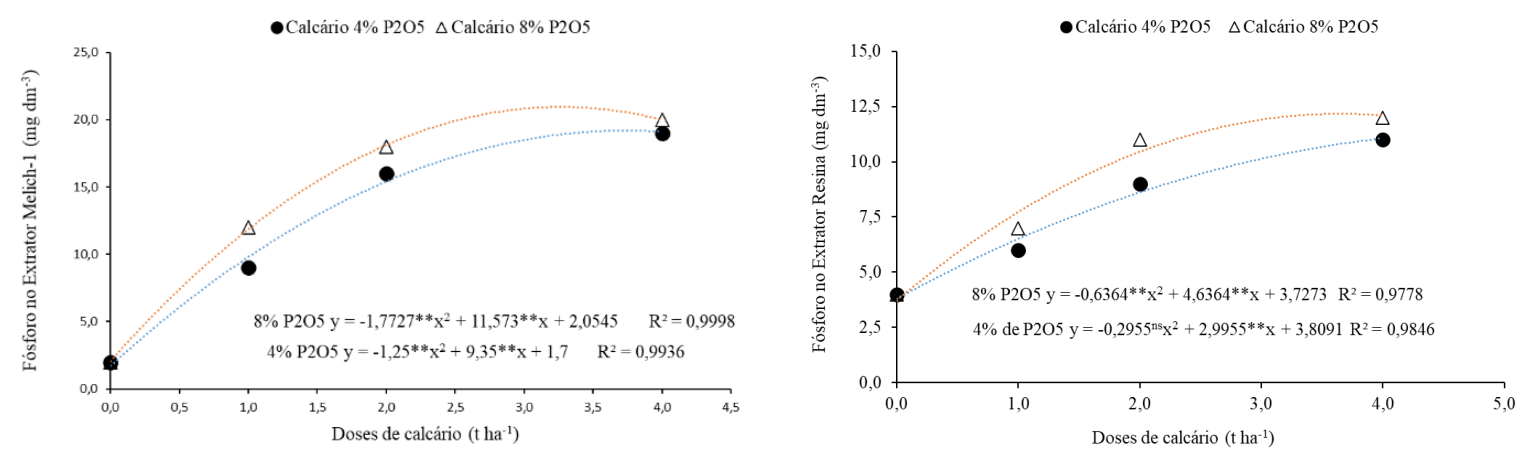

Fonte: Autores.

Quando comparamos os valores de fósforo do tratamento testemunha, nos extratores Mehlich-1 e Resina, não houve diferença signficitaiva entre eles. No entanto, ao compararmos os teores de fósforo disponivel entre os extratores para os tratamentos com materiais calcários testados, verifica-se que no extrator Mehlich-1 os valores são mais elevados que os da Resina. Este fato pode estar associado ao tipo de extrator, no caso o Mehlich-1, também conhecido como o $(0,05 \mathrm{HCl}+0,025$ $\mathrm{H}_{2} \mathrm{SO}_{4}$ ) ou Carolina do Norte poderia estar solubilizando parte do fósforo do material calcário e superestimando o fósforo disponível para as plantas. Como a Resina é um extrator neutro, seria mais indicado para monitorar este nutriente no solo, para este tipo de material. Freitas (2012) comenta que o extrator Mehlich-1, baseia-se no princípio da dissolução ácida de fósforo ligado ao cálcio ou de fosfatos naturais de modo geral, e a dessorção do P retido nas superfícies de coloides do solo pelo ânion sulfato. Assim, o extrator Mehlich-1 é, consensualmente, recomendado para solos ácidos e de CTC baixa, que não tenham recebido fosfatos naturais, como no caso de solos de Cerrado. Este fato é confirmado neste trabalho onde o extrator Mehlich-1 estimou mais fósforo disponível do a resina trocadora de aníons. Alcântara, et al. (2008), relatam que solos ricos em fósforo ligados a cálcio os extratores Resina de Troca Aniônica ou Mista, o Bray-1 e o Mehlich-3, com baixa capacidade de extração de P-Ca, deverão ser mais eficientes que o Mehlich-1, o que pode ser confirmado neste estudo com o extrator Resina extraindo menor quantidade de fósforo disponível as plantas.

Os teores de cálcio foram alterados pelos calcários, tendo sua máxima resposta com 3,35 e 3,29 tha-1 para os calcários com $4 \%$ e $8 \%$ de $\mathrm{P}_{2} \mathrm{O}_{5}$, com elevação dos teores de $\mathrm{Ca}$ até 3,01 e 3,4 $\mathrm{cmol} \mathrm{dm}^{-3}$, respectivamente. Os materiais testados dobraram os teores de Ca trocável no solo e triplicaram o Mg trocável, podendo ser utilizado, além de corretivo, como fontes de Ca e $\mathrm{Mg}$ (Figura 6). Aqui também não ocorreu diferenças signficativas entre os calcários. 
Figura 6. Cálcio e magnésio trocável no solo, com doses de calcário fosfático. * e ** significativo a 5\% e $1 \%$ de probabilidade, respectivamente. ${ }^{\mathrm{ns}}$ não significativo.
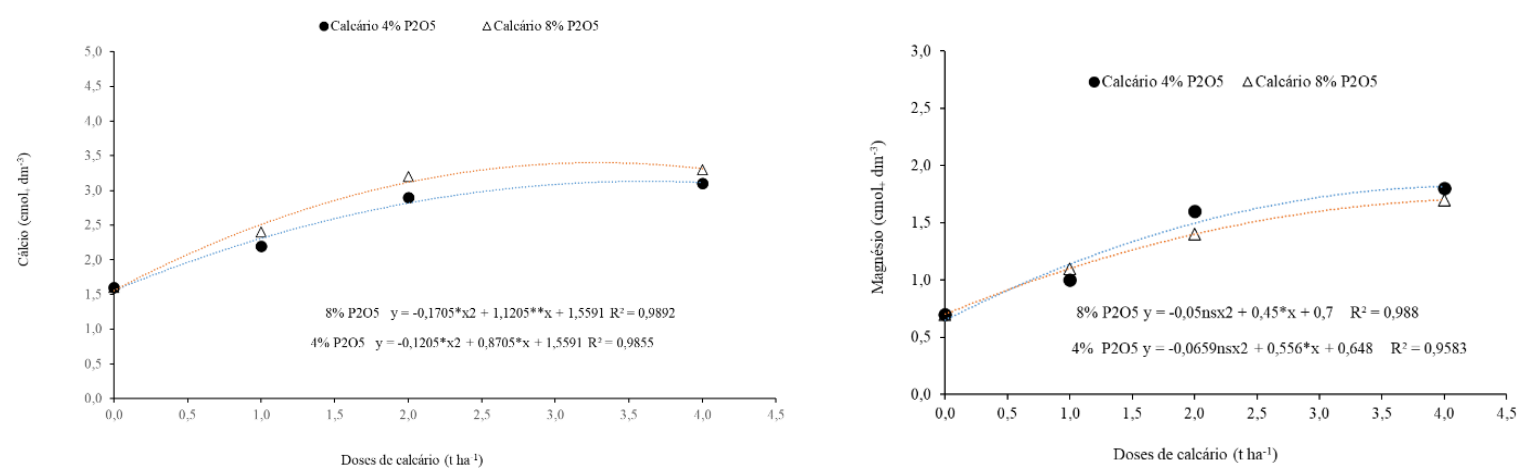

Fonte: Autores.

Quanto ao magnésio, os modelos matemáticos ajustados foram lineares, indicando que os materiais estariam ainda liberando $\mathrm{Mg}$ para a solução do solo. Pelo coeficiente angular da reta, o calcário de $4 \%$ de $\mathrm{P}_{2} \mathrm{O}_{5}$, estaria com maior velocidade de liberação de magnésio $(\mathrm{Mg})$ para a solução do solo. Este fato poderia esatr indicando que os Cabonatos de Mg seria mais solúveis que os de Ca. A solubidade do carbonato de cálcio é de $14 \mathrm{mg} \mathrm{L}^{-1}$. Se comparamos o produto de solubilidade (Kps) do $\mathrm{CaCO}_{3} 5 \times 10^{-9}$ e do $\mathrm{MgCO}_{3}$ e de 6,8 × $10^{-6}$ a $25^{\circ} \mathrm{C}$, verifica-se que o do $\mathrm{Mg}$ é mais baixo e o Kps está relacionado diretamente a solubilidade podemos dizer que os cabonatos de magnésio são mais solúveis do que de cálcio Mello \& Perez (2009), o que estaria sendo comprovado pelo resultados deste estudo. Outro aspecto que reforça a maior solubilidade carbonato de $\mathrm{Mg}$ em aos relação ao de $\mathrm{Ca}$ estaria na relação $\mathrm{Ca} / \mathrm{Mg}$, diminuindo com aumento das doses de calcário (Figura 7).

Figura 7. Valores médios da relação $\mathrm{Ca} / \mathrm{Mg}$, com doses de calcário fosfático. * e ** significativo a $5 \%$ e $1 \%$ de probabilidade, respectivamente.

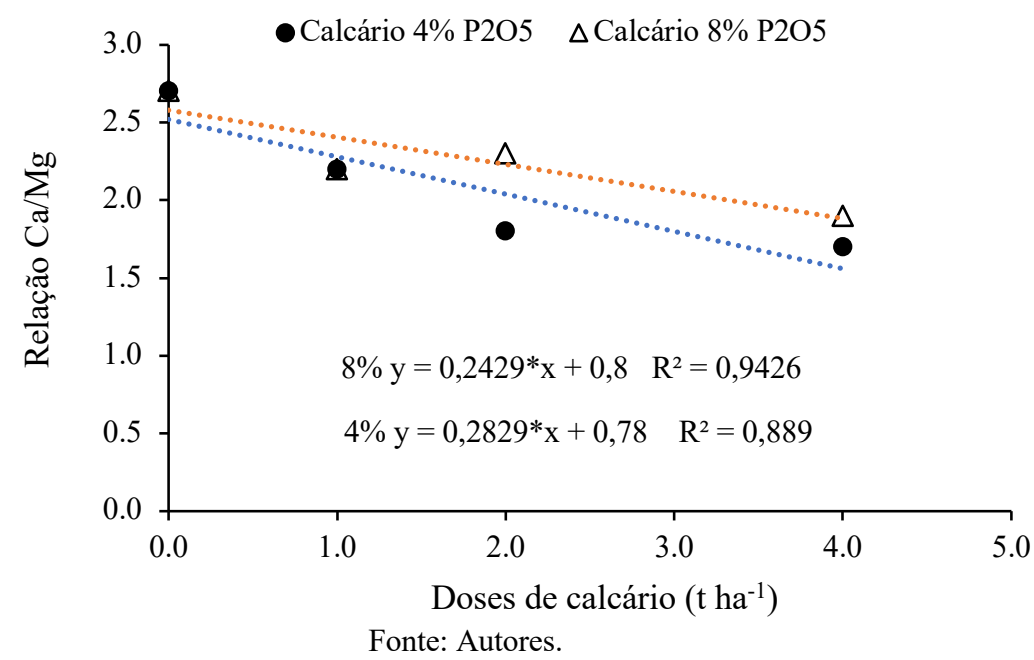

$\mathrm{Na}$ comapração relativa da capacidade de neutralização o $\mathrm{MgCO}_{3}$ tem valor de 1,19 em relação ao valor de 1,0 do $\mathrm{CaCO}_{3}$.

Considerando o modelo e o intervalo das doses estudadas, os teores máximos de $\mathrm{Mg}$ liberados seriam de 1,91 e 1,77 $\mathrm{cmol}^{-3}$ de magnésio $(\mathrm{Mg})$ trocável para o solo. Assim como o $\mathrm{Ca}$, os calcários não apresentaram diferenças entre eles e elevaram os 
teores no solo de $0,65 \mathrm{cmol} \mathrm{dm}^{-3}$ (Testemunha) para 1,84 $\mathrm{cmol} \mathrm{dm}^{3}$, teor médio dos materiais estudados, indicando potencial como corretivos dos teores de magnésio no solo.

\section{Conclusão}

Os materiais testados foram eficientes como corretivos da acidez do solo, dos níveis de cálcio e magnésio trocáveis no solo;

Os calcários aumentaram os teores de fosforo disponível as plantas no solo, podendo ser indicado, para fosfatagem do solo como fonte fosforo natural reativo.

\section{Referências}

Alcântara, F. A., Furtini Neto, A. E., Curi, N. \& Resende, A. V. (2008). Extraction methods for phosphorus and their relationship with soils phosphorus-buffer capacity estimated by the remaining-phosphorus methodology - A pot study with maize. Communications in Soil Science Plant Analisys, 39(3), 603-615. https://doi.org/10.1080/00103620701828395.

Alvarez V, V. H., Novais, R. F., Barros, N. F., Cantarutti, R. B. \& Lopes, A. S. (1999). Interpretação dos resultados das análises de solos. In: Ribeiro, A. C., Guimarães, P. T. G. \& Alvarez V, V. H. (Eds). Recomendações para o uso de corretivos e fertilizantes em Minas Gerais, $5^{\mathrm{a}}$ Aproximação. Viçosa: Comissão de Fertilidade do Solo do Estado de Minas Gerais.

Aquino, G. S., Medina, C. C., Porteira Junior, A. L., Santos, L. O., Cunha, A. C. B., Kussaba, D. A. O., Santos Júnior, J. H., Almeida, L. F. \& Santiafo, A. D. (2015). Sistema radicular e produtividade de soqueiras de cana-de-açúcar sob diferentes quantidades de palhada. Pesquisa Agropecuaria Brasileira, 50(12), 1150-1159. http://dx.doi.org/10.1590/S0100-204X2015001200004

Brasil. Manual de métodos analíticos oficiais para fertilizantes minerais, orgânicos, organominerais e corretivos. (2014). Ministério da Agricultura, Pecuária e Abastecimento.

Bambolim, A., Caion, E. G., Souza, N. F., Junior, G. D. F. S. \& Ferbonink, G. F. (2015). Calcário líquido e calcário convencional na correção da acidez do solo. Journal of Neotropical Agriculture, 2(3), 34-38. https://doi.org/10.32404/rean.v2i3.277

Cross, A. F. \& Schlesinger, W. H. (1995). A literature review and evaluation of the Hedley fractionation: Applications to the biogeochemical cycle of soil phosphorus in natural ecosystems. Geoderma, 64(3),197-214. https://doi.org/10.1016/0016-7061(94)00023-4.

Embrapa, Empresa Brasileira de Pesquisa Agropecuária. (2009). Manual de análises químicas de solos, plantas e fertilizantes. (2a ed.), Embrapa Solos.

Embrapa, Empresa Brasileira de Pesquisa Agropecuária. (2011). Manual de métodos de análise de solos. Embrapa Solos.

Ferraz, R. L. S.; Barbosa, M. A., Batista, J. L., Magalhaes, I. D., Dantas, G. F. \& Franco, F. O. (2015). Calagem em cana-de-açúcar: Efeitos no solo, planta e reflexos na produção. InterfacEHS 10. Saúde, Meio Ambiente e Sustentabilidade, 10(1), 166-177. https://doi.org/10.13140/RG.2.1.3830.1282

Freitas, I. F. (2012). Fósforo extraído com resinas trocadoras de íons emehlich-1 de latossolos submetidos a fontes e doses de fósforo e tempos de contato, Viçosa: Dissertação de Mestrado na Universidade Federal de Viçosa.

Jadoski, C. J., Toppa, E. V. B., Julianetti, A., Hulsbof, T. \& Ono, E. O. (2010). Physiology development in the vegetative stage of sugarcane. Pesquisa Aplicada \& Agrotecnologia, 3(2), 169-176. https://doi.org/10.5777/paet.v3i2.1064

Justo, L. J. E. C. (1999). Fosfato da Serra da Bodoquena - Mato Grosso do Sul. CPRM.

Lopes, A. (1984). Solos "sob condições de cerrados": características, propriedades e manejo. Piracicaba: Associação Brasileira para Pesquisa de Potassa e do Fosfato.

Mello, J. W. V. \& Perez, D. V. (2009). Equilíbrio químico das reações o solo. In: Melo, V. F. \& Alleoni, L. R. F. Ed. Química e mineralogia do solo, Sociedade Brasileira de Ciência do Solo.

Pavinato, P. C. \& Rosolem, C. A. (2008). Disponibilidade de nutrientes no solo decomposição e liberação de compostos orgânicos de resíduos vegetais. Revista Brasileira de Ciência do Solo, 32(3), 911- 920. https://doi.org/ 10.1590/S0100-06832008000300001

Rheinheimer, D. S. \& Anghinoni, I. (2006). Accumulation of soil organic phosphorus by soil tillage and cropping systems in subtropical soils. Communications in Soil Science and Plant Analysis, 34(15), 2339-2354. https://doi.org/10.1081/CSS-120024068.

Rheinheimer, D. S. \& Anghinoni, I. (2001). Distribuição do fósforo inorgânico em sistemas de manejo de solo. Pesquisa Agropecuária Brasileira, 36(1), 151160. https://doi.org/10.1590/S0100-204X2001000100019.

Silva, M. A., Jeronimo, E. M. \& Lucio, A. D. (2008). Perfilhamento e produtividade de cana-de-açúcar com diferentes alturas de corte e épocas de colheita, Pesquisa Agropecuária Brasileira, 43(8), 979-986. https://doi.org/10.1590/S0100-204X2008000800005.

Silva, A. M. S., Oliveira, E. C. A., Willadino, L. G., Freire, F. J. \& Rocha, A. T. (2019). Corrective phosphate application as a practice for reducing oxidative stress and increasing productivity in sugarcane. Revista Ciência Agronômica, 50(2), 188-196. https://dx.doi.org/10.5935/1806-6690.20190022. 
Research, Society and Development, v. 10, n. 7, e49210716762, 2021

(CC BY 4.0) | ISSN 2525-3409 | DOI: http://dx.doi.org/10.33448/rsd-v10i7.16762

Silveira Junior, E. G., Penatti, C., Korndörfer, G. H. \& Camargo, M. S. (2003). Silicato de cálcio e calcário na produção e qualidade da cana-de-açúcar - usina Catanduva. In: Congresso Brasileiro de Ciência do Solo, 24, 2003, Ribeirão Preto. Anais... Ribeirão Preto: Sociedade Brasileira de Ciência do Solo.

Soratto, R. P., Crusciol, C. A. C. \& Mello, F. F. C. (2010). Componentes da produção e produtividade de cultivares de arroz e feijão em função de calcário e gesso aplicados na superfície do solo. Bragantia, 69(4), 965-974. https://doi.org/10.1590/S0006-87052010000400023.

Souza, D. M. G., Miranda, L. N. \& Oliveira, S. A. (2007) Acidez do solo e sua correção. In: Novais, R. F., Barros, N. F. de, Fontes, L. F., Cantarutti, R. B. \& Lima, J. C., Fertilidade do solo, Viçosa: Sociedade Brasileira de Ciência do Solo.

Souza, R. M., Sobral, L. F. Viégas, P. R. A., Oliveira Junior, A. \& Carvalho, M. C. S. (2014). Eficiência agronômica de fosfatos de rocha em solo com elevado teor de cálcio trocável. Revista Brasileira de Ciência do Solo, 38(6), 1816-1825. https://dx.doi.org/10.1590/S0100-06832014000600016.

Tavares, O. C., Huertas, L. E. \& Zonta, E. (2010). Crescimento e produtividade da cana planta cultivada em diferentes sistemas de preparo do solo e de colheita. Acta Scientiarum. Agronomy, 32(1),61-68. https://doi.org/10.4025/actasciagron.v32i1.205110.4025/actasciagron.v32i1.2051

Vitti, G. C., Lima, E. \& Cicarone, F. (2006) Cálcio, Magnésio e Enxofre. In: Nutrição Mineral de Plantas. Sociedade Brasileira de Ciência do Solo.

Zandoná, R. R., Beutler, A. N., Burg, G. M., Barreto, C. F. \& Schmidt, M. R. (2015). Gesso e calcário aumentam a produtividade e amenizam o efeito do déficit hídrico em milho e soja. Pesquisa Agropecuária Tropical, 45(2), 128-137. https://doi.org/10.1590/1983-40632015v4530301.

Walker, T. W. \& Syers, J. K. (1976). The fate of phosphorus during pedogenesis. Geoderma, 15(1),1-19. https://doi.org/10.1016/0016-7061(76)90066-5. 